\title{
Streptococcus salivarius
}

National Cancer Institute

\section{Source}

National Cancer Institute. Streptococcus salivarius. NCI Thesaurus. Code C86804.

A species of facultatively anaerobic, Gram positive, cocci shaped bacteria in the phylum Firmicutes. This species is alpha hemolytic, hydrolyzes esculin and is negative for arginine deaminase, and catalase. It can ferment inulin, salicin, and raffinose but not mannitol, or sorbitol. S. salivarius is found in the normal flora of the mouth and respiratory tract and is rarely a human pathogen causing opportunistic infections. 